Bulletin of the Section of Logic

Volume 45:3/4 (2016), pp. 185-197

http://dx.doi.org/10.18778/0138-0680.45.3.4.04

Marcin Lazarz

\title{
CHARACTERIZATION OF BIRKHOFF'S CONDITIONS BY MEANS OF COVER-PRESERVING AND PARTIALLY COVER-PRESERVING SUBLATTICES
}

\begin{abstract}
In the paper we investigate Birkhoff's conditions $(\mathrm{Bi})$ and $\left(\mathrm{Bi}^{*}\right)$. We prove that a discrete lattice $L$ satisfies the condition $(\mathrm{Bi})$ (the condition $\left(\mathrm{Bi}^{*}\right)$ ) if and only if $L$ is a 4-cell lattice not containing a cover-preserving sublattice isomorphic to the lattice $S_{7}^{*}$ (the lattice $S_{7}$ ). As a corollary we obtain a well known result of J. Jakubík from [6].

Furthermore, lattices $S_{7}$ and $S_{7}^{*}$ are considered as so-called partially coverpreserving sublattices of a given lattice $L, S_{7} \ll L$ and $S_{7}^{*} \ll L$, in symbols. It is shown that an upper continuous lattice $L$ satisfies $\left(\mathrm{Bi}^{*}\right)$ if and only if $L$ is a 4 -cell lattice such that $S_{7} \nless L$. The final corollary is a generalization of Jakubík's theorem for upper continuous and strongly atomic lattices.

Keywords: Birkhoff's conditions, semimodularity conditions, modular lattice, discrete lattices, upper continuous lattice, strongly atomic lattice, cover-preserving sublattice, cell, 4-cell lattice.
\end{abstract}

\section{Preliminaries}

Standard lattice-theoretic notions can be found in [4]. For the reader's convenience we will recall basic notions and facts. Let $L$ be an arbitrary lattice. If $x, y \in L$ such that $x \leq y$, then the set $[x, y]_{L}=\{z \in L: x \leq$ $z \leq y\}$ is called an interval in $L$. If $L$ is fixed, we write $[x, y]$ instead of $[x, y]_{L}$. Clearly, any interval in $L$ is a sublattice of $L$. Moreover, let $\langle x, y\rangle=[x, y] \backslash\{x, y\}$. If $\langle x, y\rangle=\emptyset$ we then write $x \prec y$ ( $x$ is covered by $y)$. 
A lattice $C$ is called a cell (see $[9,8]$ ) if and only if there are two different points $v, u \in C$ and chains $C_{1}, C_{2} \subseteq C$ such that:

(a) $C_{1} \cap C_{2}=\{v, u\}, \quad C=C_{1} \cup C_{2} \neq\{v, u\}$,

(b) $\left(\forall x \in C_{1} \backslash\{v, u\}\right)\left(\forall y \in C_{2} \backslash\{v, u\}\right)(x \vee y=u \quad \& \quad x \wedge y=v)$.

Finite cells are denoted by $C_{m, n}$ (see Figure 1). Obviously $C_{m, n}$ and $C_{n, m}$ are isomorphic. The lattice $C_{3,3}$, usually denoted by $B_{2}$, is the fourelement Boolean lattice. We call it an improper cell; the remaining cells are said to be proper. The proper cell $C_{3,4}$ is called the pentagon and it is denoted by $N_{5}$.
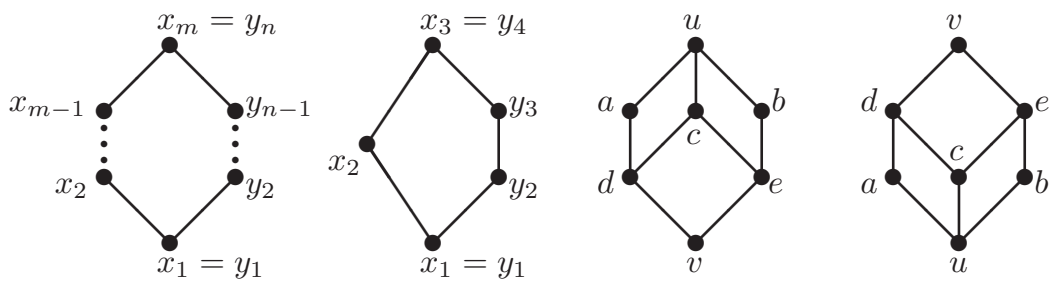

Fig. 1. From left to right: lattices $C_{m, n}, C_{3,4}\left(\right.$ i.e. $\left.N_{5}\right), S_{7}$ and $S_{7}^{*}$.

We say that $C$ is a cell sublattice of a lattice $L$ if

(c) $C$ is a sublattice of $L$,

(d) $C$ is a cell as a lattice,

(e) chains $C_{1}, C_{2}$ are maximal in the interval $[v, u]_{L}$.

If a lattice $L$ has the following property:

every cell of $L$ is isomorphic to $B_{2}$,

(4-cell)

we then say that $L$ is a 4 -cell lattice (see [5]). The examples of 4-cell lattices are: $B_{2}, S_{7}, S_{7}^{*}$, the first and the second lattice presented in Figure 6 .

We write $M<L$ if $M$ is a sublattice of $L$. A sublattice $M$ of a lattice $L$ is called cover-preserving (see [3]), $M \prec L$ in symbols, if $x \prec y$ in $M$ implies $x \prec y$ in $L$, for all $x, y \in M$. For instance, $N_{5}$ is a sublattice, but it is not a cover-preserving sublattice of $S_{7}$. It is also clear that cell sublattices are cover-preserving sublattices.

For purposes of our research we consider lattices $S_{7}$ and $S_{7}^{*}$ as "partially cover-preserving sublattices": we write $S_{7} \ll L$ if $a \prec u$ and $b \prec u$ in $L$ 
(see the second picture in Figure 2, where double lines denote the covering relation), and dually, $S_{7}^{*} \ll L$ if $u \prec a$ and $u \prec b$ in $L$.
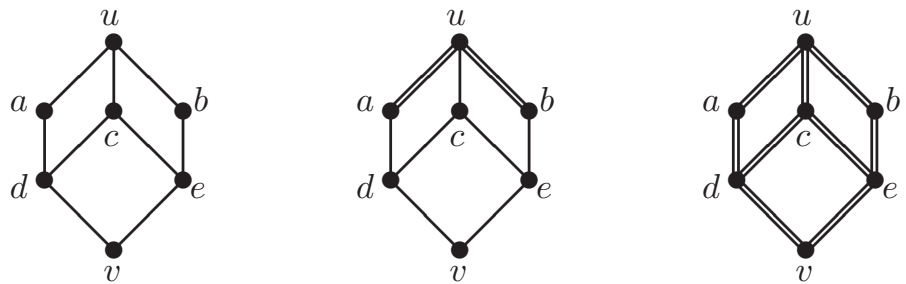

Fig. 2. From left to right: $S_{7}<L, S_{7} \ll L$ and $S_{7} \prec L$.

A lattice $L$ is said to be modular if $x \leq z$ implies $x \vee(y \wedge z)=(x \vee y) \wedge z$, for all $x, y, z \in L$. Modularity is one of the most important concepts in lattice theory. It was introduced by R. Dedekind in 1900 as a property of the structure of all normal subgroups of a given group (see $[1, \S 9.6]$ ). The famous characterization given by Dedekind states that a lattice $L$ is modular if and only if $L$ does not contain a sublattice isomorphic to $N_{5}$.

A number of conditions weaker than modularity are considered in the literature (see $[11,10])$. In this paper we are focused on so-called Birkhoff's conditions

$$
\begin{aligned}
& (\forall x, y \in L)(x \wedge y \prec x, y \Rightarrow x, y \prec x \vee y), \\
& (\forall x, y \in L)(x, y \prec x \vee y \Rightarrow x \wedge y \prec x, y),
\end{aligned}
$$

and closely related to them semimodularity conditions

$$
\begin{aligned}
& (\forall x, y \in L)(x \wedge y \prec x \Rightarrow y \prec x \vee y), \\
& (\forall x, y \in L)(y \prec x \vee y \Rightarrow x \wedge y \prec x) .
\end{aligned}
$$

It is a trivial fact that modularity implies all preceding conditions, and moreover, $(\mathrm{Sm})$ implies $(\mathrm{Bi})$, and $\left(\mathrm{Sm}^{*}\right)$ implies $\left(\mathrm{Bi}^{*}\right)$. The converses of these implications are not true in general: the second lattice presented in Figure 6 satisfies (Bi) but violates ( $\mathrm{Sm})$. Moreover, if $L$ satisfies $(\mathrm{Bi})$, then $S_{7}^{*} \nless L$, and all the more $S_{7}^{*} \nprec L$, but not necessarily $S_{7}^{*} \nless L$ (see Figure 3). Dually, if $L$ satisfies $\left(\mathrm{Bi}^{*}\right)$, then $S_{7} \nless L$, but not necessarily $S_{7} \nless L$. It is also clear that proper cells $C_{m, n}$ violate both Birkhoff's conditions. 
For finite lattices we have the following classical result:

Proposition 1. If $L$ is a finite lattice, then:

(i) (Sm) is equivalent to (Bi), and $\left(S m^{*}\right)$ is equivalent to $\left(B i^{*}\right)$,

(ii) modularity is equivalent to the conjunction of $(\mathrm{Sm})$ and $\left(\mathrm{Sm}^{*}\right)$,

(iii) modularity is equivalent to the conjunction of $(\mathrm{Bi})$ and $\left(B i^{*}\right)$.

For the proof see [4, Chapter IV.2] or [12, Chapter 19]. Since the proof of Proposition 1 is inductive, the analogous theorem is true for lattices of finite length (i.e. there is a natural number $n$ such that every chain has at most $n$ elements) and discrete $^{1}$ ones (i.e. every bounded chain is finite).

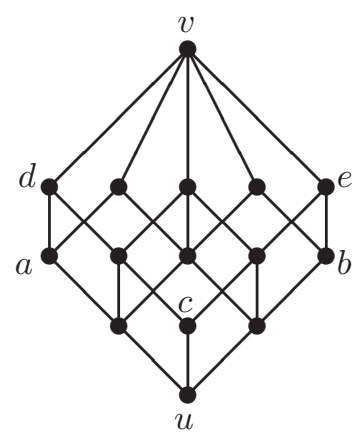

Fig. 3. The 4-cell lattice which satisfies (Bi) and contains a sublattice $S_{7}^{*}$.

An important extension of Proposition 1 requires further notions. A lattice $L$ is called upper continuous if $L$ is complete and for every element $x \in L$ and every chain $C \subseteq L$ holds

$$
x \wedge \bigvee C=\bigvee\{x \wedge c: c \in C\} .
$$

A lattice $L$ is called strongly atomic if

$$
(\forall x, y \in L)(x<y \Rightarrow(\exists z \in L)(x \prec z \leq y)) .
$$

Lattices of finite length are strongly atomic and upper continuous. On the other hand, discrete lattices are strongly atomic, and complete discrete lattices are upper continuous. Moreover, (UC) is a generalization of the

\footnotetext{
${ }^{1}$ Some authors use the term "a lattice of locally finite length", see $[11$, p. 60] or [6].
} 
ascending chain condition, ACC (i.e. there is no infinite ascending chain), and (SA) is a generalization of the descending chain condition, DCC (i.e. there is no infinite descending chain).

Proposition 2. If $L$ is an upper continuous and strongly atomic lattice, then:

(i) $([2$, Theorem 3.7]) (Sm) is equivalent to (Bi),

(ii) $([2$, Theorem 3.6]) modularity is equivalent to the conjunction of (Sm) and $\left(\mathrm{Sm}^{*}\right)$,

(iii) ([7, Proposition 4]) modularity is equivalent to the conjunction of (Bi) and $\left(B i^{*}\right)$.

Although the preceding theorems (i) and (ii) are originally formulated for compactly generated (i.e. algebraic) and strongly atomic lattices, their proofs use upper continuity and strong atomicity only (see [11, p. 39]). Note also that upper continuity and strong atomicity do not imply algebraicity (see [10, p. 338]).

Problem 1. Do $(U C),(S A)$ and $\left(B i^{*}\right)$ imply $\left(S m^{*}\right)$ ?

\section{Birkhoff's conditions in discrete lattices}

THEOREM 1. If $L$ is a discrete lattice, then the following conditions are equivalent:

(i) L satisfies (Bi),

(ii) $L$ is a 4-cell lattice and $S_{7}^{*} \nprec L$.

Proof: (i) $\Rightarrow$ (ii). The implication is trivial.

(ii) $\Rightarrow$ (i). Assume that $L$ is a 4 -cell lattice such that $S_{7}^{*} \nprec L$, and suppose to the contrary that there are $a, b \in L$ which violate the condition (Bi). Since $L$ is discrete, the induction principle allows us to assume that every proper interval of $[a \wedge b, a \vee b]$ satisfies (Bi). Put $u=a \wedge b, v=a \vee b$, and without loss of generality assume that $a \nprec v$. Then there is $d \in L$ such that $a<d \prec v$, and clearly

$$
d \wedge b=u \text {. }
$$

Now observe that $b \nprec v$. Indeed, if $b \prec v$, then taking arbitrary chain $C$ maximal in $[a, d]$, by (2.1), we achieve a proper cell $\{u, a, b, d, v\} \cup C$; 
a contradiction. Therefore, there exists $e \in L$ such that $b<e \prec v$, and similarly as before:

$$
e \wedge a=u
$$

Put $c=d \wedge e$ and note that $c>u$ (see the first picure in Figure 4).
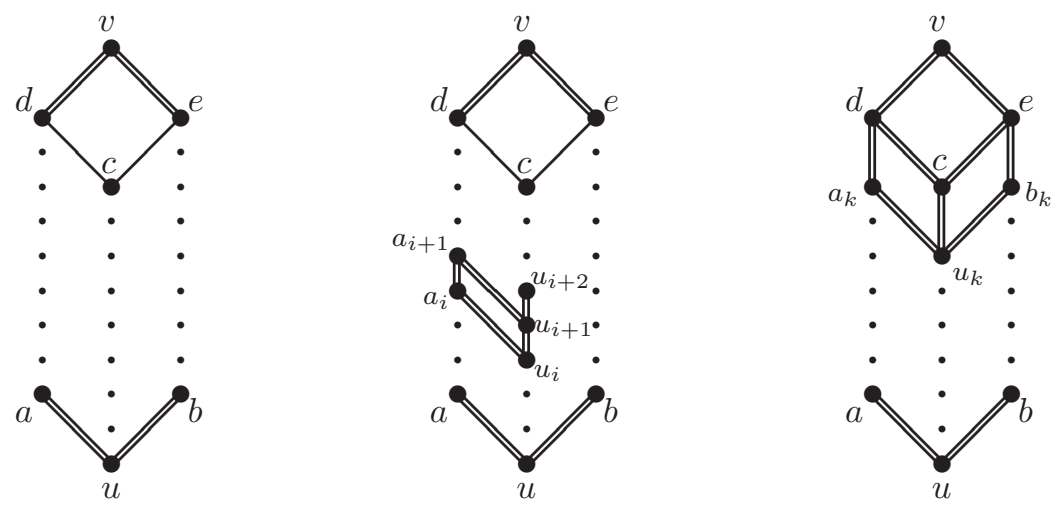

Fig. 4. The illustration of the proof of Theorem 1.

By our general assumption, the interval $[u, c]$ contains only finite chains. Fix a maximal one such that:

$$
u=u_{0} \prec u_{1} \prec u_{2} \prec \ldots \prec u_{k} \prec u_{k+1}=c,
$$

and moreover define $a_{i}=a \vee u_{i}$ for $i=0, \ldots, k+1$. By induction on $i$ we will show that:

$$
u_{i}=a_{i} \wedge u_{i+1} \prec a_{i}, u_{i+1} .
$$

For $i=0$ we have $u_{0}=u, a_{0}=a$, so we easily get $u_{0}=a_{0} \wedge u_{1} \prec a_{0}, u_{1}$.

As the induction hypothesis, assume (2.3) (see the second picture in Figure 4$)$. Since the interval $[u, d]$ is properly contained in $[u, v]$, it satisfies (Bi), therefore we obtain $a_{i}, u_{i+1} \prec a_{i} \vee u_{i+1}=a_{i+1}$. In particular, since $u_{i+1} \prec a_{i+1}$ we achieve $a_{i+1} \wedge u_{i+2}=u_{i+1}$, so finally

$$
u_{i+1}=a_{i+1} \wedge u_{i+2} \prec a_{i+1}, u_{i+2},
$$

and the proof of (2.3) is complete. 
By (2.3), for $i=k$, we have $u_{k}=a_{k} \wedge c \prec a_{k}, c$, therefore once again by $(\mathrm{Bi})$ we get

$$
a_{k}, c \prec a_{k} \vee c .
$$

Similarly defining $b_{i}=b \vee u_{i}$ and using the parallel argument we achieve

$$
u_{k}=b_{k} \wedge c \prec b_{k}, c \quad \text { and } \quad b_{k}, c \prec b_{k} \vee c .
$$

Now, by (4-cell) it follows that $a_{k} \vee c=d$ and $b_{k} \vee c=e$ (see the third picture in Figure 4), and therefore, $S_{7}^{*} \cong\left\{u_{k}, a_{k}, c, b_{k}, d, e, v\right\} \prec L$. This contradiction completes the proof.

The proof of Theorem 1 can be easily dualized, therefore we get:

THEOREM 2. If $L$ is a discrete lattice, then the following conditions are equivalent:

(i) L satisfies $\left(B i^{*}\right)$,

(ii) $L$ is a 4-cell lattice and $S_{7} \nprec L$.

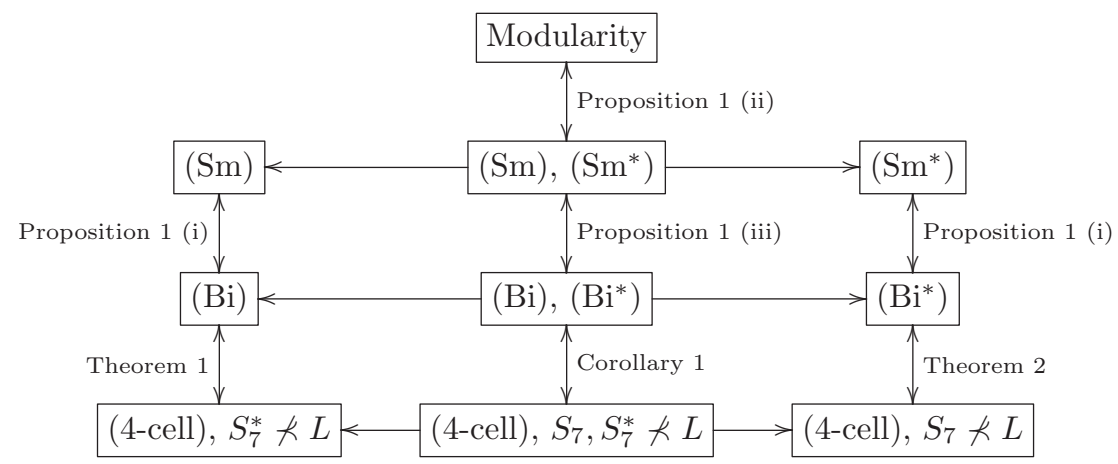

Fig. 5. The considered conditions in the class of discrete lattices.

As a corollary of Theorems 1 and 2 we achieve a result of J. Jakubík:

Corollary 1 ([6]). If $L$ is a discrete lattice, then the following conditions are equivalent: 
(i) $L$ is a modular lattice,

(ii) $L$ is a 4-cell lattice and $S_{7} \nprec L$ and $S_{7}^{*} \nprec L .^{2}$

The interrelationships between considered conditions in the class of discrete lattices are visualized in Figure 5.

\section{Birkhoff's conditions in upper continuous and strongly atomic lattices}

In this section we are interested in a generalization of results from Section 2. Unfortunately, even in the "well behaved" class of upper continuous and strongly atomic lattices the implication (ii) $\Rightarrow$ (i) of Theorem 1 is false - the first lattice presented in Figure 6 is an appropriate counterexample.
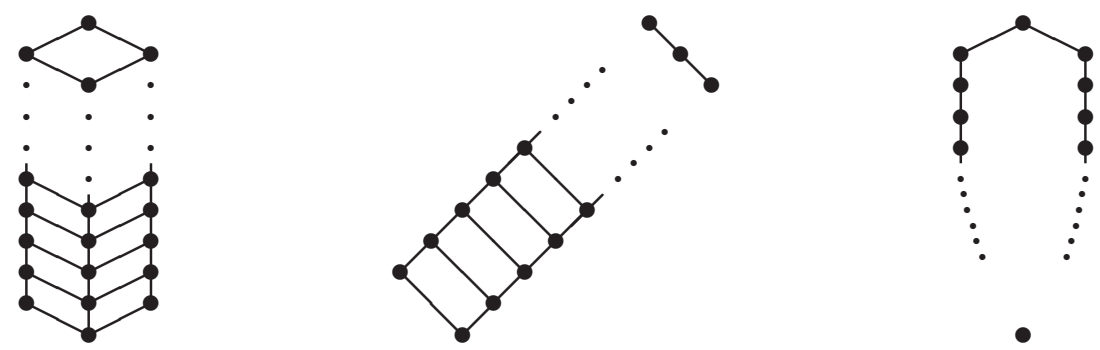

Fig. 6. From left to right: (a) The 4-cell lattice satisfying (UC), (SA), and $S_{7}^{*} \nprec L$ but violating (Bi). (b) The 4-cell lattice satisfying $S_{7}^{*} \nless L$ but violating $(\mathrm{Sm})$. (c) The lattice which satisfies $(\mathrm{Bi})$ and $(\mathrm{UC})$ but violates (4-cell).

However, replacing the assumption $S_{7}^{*} \nprec L$ by $S_{7}^{*} \nless L$, we obtain the following general theorem:

Theorem 3. If $L$ is a 4 -cell lattice and $S_{7}^{*} \nless L$, then $L$ satisfies (Bi).

Proof: Suppose to the contrary that $L$ does not satisfy (Bi), so there are $a, b \in L$ which violate (Bi). Put $u=a \wedge b$ and $v=a \vee b$; without loss of generality we assume that $\langle a, v\rangle \neq \emptyset$ (see Figure 7).

\footnotetext{
${ }^{2}$ There is a misprint in the original formulation of the theorem in [6]: instead of "sublattice" should be "c-sublattice" (i.e. "cover-preserving sublattice").
} 
By the Axiom of Choice, there exists a maximal chain $C_{1}$ in the interval $\langle a, v\rangle$. Since $u \prec b$ we easily show that:

$$
\left(\forall x \in C_{1}\right)(x \wedge b=u) .
$$

Supposing $b \prec v$ by (3.1) we obtain a proper cell $\{u, a, b, v\} \cup C_{1}$, which contradicts the assumption of the theorem. Hence $\langle b, v\rangle \neq \emptyset$, and therefore the Axiom of Choice provides that there exists a maximal and non-empty chain $C_{2}$ contained in $\langle b, v\rangle$. Clearly there are $d \in C_{1}$ and $e \in C_{2}$ such that $d \wedge e>u$ (otherwise, $\{u, a, b, v\} \cup C_{1} \cup C_{2}$ would be a proper cell).

Put $c=d \wedge e, d_{1}=a \vee c$, and $e_{1}=b \vee c$. Clearly $a<d_{1}<v$ and $b<e_{1}<v$. Now observe that

$$
d_{1} \wedge e_{1}=c \text { and } c<d_{1} \text { and } c<e_{1} .
$$

Indeed, $c \leq d_{1} \wedge e_{1}$ since $c \leq d_{1}$ and $c \leq e_{1}$. On the other hand $d_{1} \leq d$ and $e_{1} \leq e$, therefore $d_{1} \wedge e_{1} \leq d \wedge e=c$. Now supposing for instance $c=d_{1}$ we have $c=a \vee c$ i.e. $a \leq c$, so $a \vee b \leq c \vee b<v$ which is impossible.

Finally, we obtain $S_{7}^{*} \cong\left\{u, a, b, c, d_{1}, e_{1}, v\right\} \ll L-$ a contradiction.

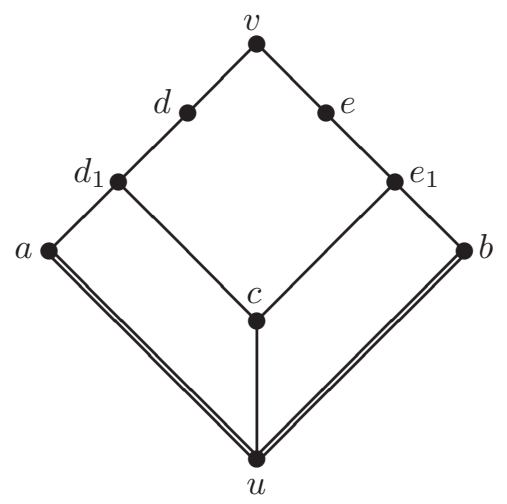

Fig. 7. The illustration of the proof of Theorem 3

Let us notice that the premises of Theorem 3 do not imply the condition $(\mathrm{Sm})$ (see the second lattice in Figure 6). Furthermore, there arises a problem under which assumptions the converse of Theorem 3 is true. Obviously, if $L$ satisfies ( $\mathrm{Bi})$, then $S_{7}^{*} \nless L$. On the other hand, the implication

$$
(\mathrm{Bi}) \Rightarrow(4 \text {-cell })
$$


which was trivially valid for discrete lattices, it turns out a difficult problem for "properly infinite" lattices.

REMARK 1.

(i) (Bi) and (UC) do not imply (4-cell).

(ii) (Bi) and (SA) do not imply (4-cell).

Proof: Ad (i). The third lattice in Figure 6 is a suitable counterexample. Ad (ii). Let $\mathbb{Z}^{*}=\mathbb{Z} \backslash\{0\}, \mathbb{Z}^{+}=\{1,2,3, \ldots\}, \mathbb{Z}^{-}=\{-1,-2,-3, \ldots\}$ and $\operatorname{Fin}\left(\mathbb{Z}^{*}\right)=\left\{X \subseteq \mathbb{Z}^{*}: X\right.$ is finite $\}$. Consider the set $L$ defined as follows:

$$
L=\mathcal{P}\left(\mathbb{Z}^{+}\right) \cup \mathcal{P}\left(\mathbb{Z}^{-}\right) \cup \operatorname{Fin}\left(\mathbb{Z}^{*}\right) \cup\left\{\mathbb{Z}^{*}\right\},
$$

and operations:

$$
X \wedge Y=X \cap Y, \quad \text { and } \quad X \vee Y=\left\{\begin{array}{ll}
X \cup Y & \text { if } X \cup Y \in L \\
\mathbb{Z}^{*} & \text { otherwise }
\end{array},\right.
$$

for all $X, Y \in L$ (see Figure 8). It is easy to see that the algebra $(L, \vee, \wedge)$ is a lattice which satisfies $(\mathrm{Bi})$ and $(\mathrm{SA})$. However it is not a 4 -cell lattice. Indeed, put $C_{n}=\mathbb{Z}^{+} \backslash\{1,2,3, \ldots, n\}$ and $D_{n}=\mathbb{Z}^{-} \backslash\{-1,-2,-3, \ldots,-n\}$, for every $n \geq 1$. Then it is easy to verify that

$$
\mathcal{C}=\left\{C_{1}, C_{2}, C_{3}, \ldots\right\}, \quad \mathcal{D}=\left\{D_{1}, D_{2}, D_{3}, \ldots\right\} .
$$

are maximal chains in $\left\langle\emptyset, \mathbb{Z}^{+}\right\rangle$and $\left\langle\emptyset, \mathbb{Z}^{-}\right\rangle$, respectively. Hence $\mathcal{C} \cup \mathcal{D} \cup$ $\left\{\emptyset, \mathbb{Z}^{*}\right\}$ is an infinite cell in $L$.

Problem 2. Do (Bi), (UC) and (SA) imply (4-cell)?

Corollary 2. If $L$ is a 4-cell lattice, then the following conditions are equivalent:

(i) L satisfies (Bi),

(ii) $S_{7}^{*} \nless L$.

Now, let us consider the condition $\left(\mathrm{Bi}^{*}\right)$. Clearly, the proof of Theorem 3 can be dualized, therefore we get: 


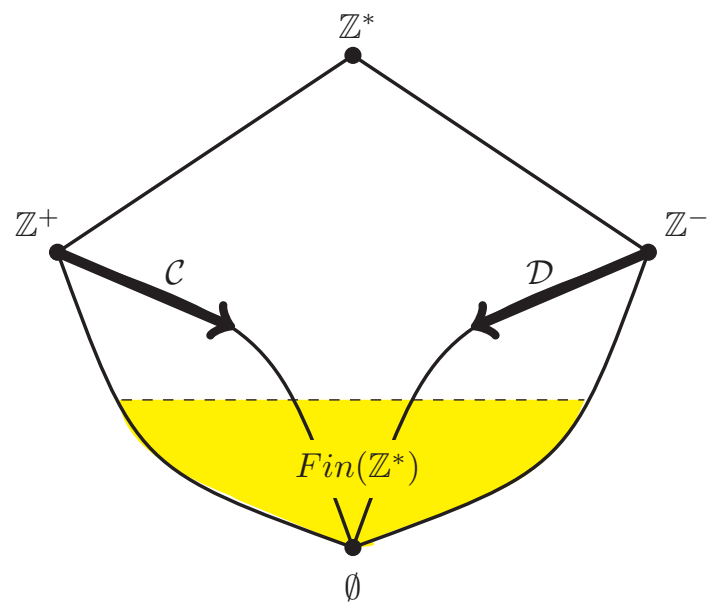

Fig. 8. A lattice which satisfies (Bi) and (SA) but violates (4-cell).

Theorem 4. If $L$ is a 4 -cell lattice and $S_{7} \nless L$, then $L$ satisfies $\left(B i^{*}\right)$.

Similarly as before, if $L$ satisfies $\left(\mathrm{Bi}^{*}\right)$, then $S_{7} \nless L$. It is also obvious that $\left(\mathrm{Bi}^{*}\right)$ does not imply (4-cell): as a counterexample consider the lattice which is dual to the third lattice presented in Figure 6. However we have: Theorem 5. (Bi $i^{*}$ and (UC) imply (4-cell).

Proof: Suppose that $C=C_{1} \cup C_{2}$ is a proper cell of $L$ with the least element $v$ and the greatest element $u$. Consider the two following cases:

Case 1. If $C_{1} \backslash\{u\}$ and $C_{1} \backslash\{u\}$ contain maximal elements, $m_{1}$ and $m_{2}$ respectively, then $m_{1}, m_{2} \prec u$, so $\left(\mathrm{Bi}^{*}\right)$ provides $v=m_{1} \wedge m_{2} \prec$ $m_{1}, m_{2}$, which means that $C$ is isomorphic to $B_{2}$-a contradiction with the hypothesis.

Case 2. Assumig that $C_{1} \backslash\{u\}$ has no maximal element, we easily prove $\bigvee\left(C_{1} \backslash\{u\}\right)=u$ (the lattice is complete since it is upper continuous). Now choosing arbitrary $a \in C_{2} \backslash\{v, u\}$ and applying (UC) we get a contradiction:

$$
a=a \wedge u=a \wedge \bigvee\left(C_{1} \backslash\{u\}\right)=\bigvee\left\{a \wedge x: x \in C_{1} \backslash\{u\}\right\}=v
$$


Corollary 3. If $L$ is an upper continuous lattice, then the following conditions are equivalent:

(i) L satisfies $\left(B i^{*}\right)$,

(ii) $L$ is a 4-cell lattice and $S_{7} \nless L$.

Corollary 4. If $L$ is an upper continuous and strongly atomic lattice, then the following conditions are equivalent:

(i) $L$ is modular,

(ii) $L$ is a 4-cell lattice and $S_{7} \nless L$ and $S_{7}^{*} \nless L$.

Proof: (i) $\Rightarrow$ (ii). The implication is trivial.

(ii) $\Rightarrow$ (i). By assumptions and Theorems 3 and 4 , the lattice $L$ satisfies (Bi) and $\left(\mathrm{Bi}^{*}\right)$, therefore by Proposition 2 (iii) $L$ is modular.

The interrelationships between considered conditions in the class of upper continuous and strongly atomic lattices are visualized in Figure 9.

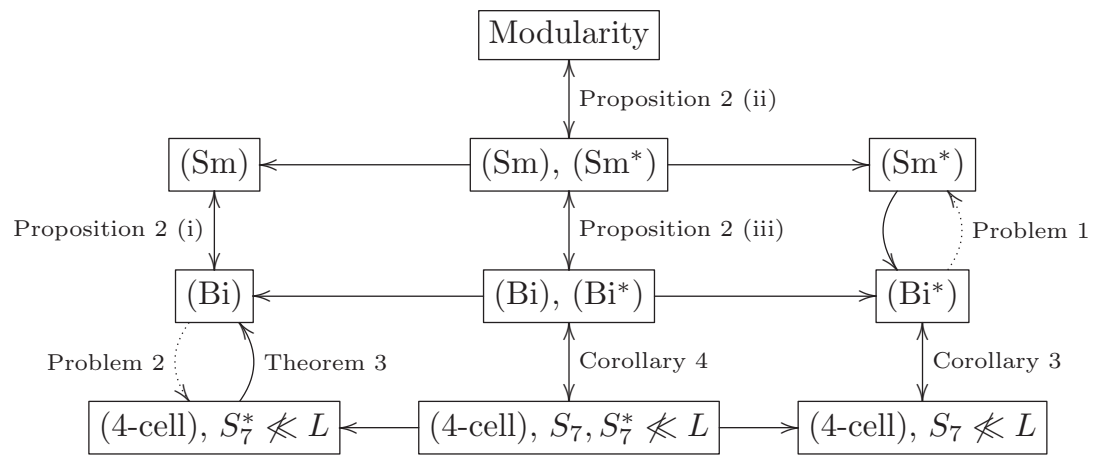

Fig. 9. The considered conditions in the class of upper continuous and stronly atomic lattices.

Acknowledgement. I am grateful to anonymous referees for their important remarks. 


\section{References}

[1] G. Birkhoff, T.C. Bartee, Modern applied algebra, McGraw-Hill Book Company XII, New York etc. (1970).

[2] P. Crawley, R.P. Dilworth, Algebraic theory of lattices, Prentice-Hall, Inc., Englewood Cliffs, New Jersey (1973).

[3] E. Fried, G. Grätzer, H. Lakser, Projective geometries as cover-preserving sublattices, Algebra Universalis 27 (1990), pp. 270-278.

[4] G. Grätzer, General lattice theory, Birkhäuser, Basel, Stuttgart (1978).

[5] G. Grätzer, E. Knapp, Notes on planar semimodular lattices. I: Construction, Acta Sci. Math. 73, No. 3-4 (2007), pp. 445-462.

[6] J. Jakubík, Modular lattice of locally finite length, Acta Sci. Math. 37 (1975), pp. 79-82.

[7] M. Łazarz, K. Siemieńczuk, Modularity for upper continuous and strongly atomic lattices Algebra Universalis 76 (2016), pp. 493-95.

[8] S. MacLane, A conjecture of Ore on chains in partially ordered sets, Bull. Am. Math. Soc. 49 (1943), pp. 567-568.

[9] O. Ore, Chains in partially ordered sets, Bull. Am. Math. Soc. 49 (1943), pp. 558-566.

[10] M. Ramalho, On upper continuous and semimodular lattices, Algebra Universalis 32 (1994), pp. 330-340.

[11] M. Stern, Semimodular Lattices. Theory and Applications, Cambridge University Press (1999).

[12] A. Walendziak, Podstawy algebry ogólnej i teorii krat, Wydawnictwo Naukowe PWN, Warszawa (2009).

Department of Logic and Methodology of Sciences

University of Wrocław, Poland

lazarzmarcin@poczta.onet.pl 